\title{
A slow bar in a dark matter dominated galaxy ${ }^{\star}$
}

\author{
L. Chemin ${ }^{1}$ and O. Hernandez ${ }^{2}$ \\ ${ }^{1}$ GÉPI, Observatoire de Paris, section Meudon, CNRS \& Université Paris 7 UMR 8111, 5 pl. Janssen, 92195 Meudon, France \\ e-mail: laurent . chemin@obspm. fr \\ 2 Université de Montréal, Département de Physique, succ. centre-ville, Montréal (QC), H3C3J7, Canada \\ e-mail: olivier@astro.umontreal.ca
}

Received 9 March 2009 / Accepted 22 April 2009

ABSTRACT

\begin{abstract}
Aims. We report on an estimate of the bar pattern speed $\Omega_{\mathrm{p}}$ for the low surface brightness spiral galaxy UGC 628 .
Methods. We applied the Tremaine-Weinberg method to high resolution $\mathrm{H} \alpha$ velocity and integrated emission maps of this dark matter dominated galaxy. Observations were made at the CFHT using the optical Fabry-Perot interferometer, FANT0мM.

Results. The Tremaine-Weinberg method estimates a bar pattern speed of $(11.3 \pm 2.0) \mathrm{km} \mathrm{s}^{-1} \mathrm{kpc}^{-1}$ for UGC 628 , which is among the lowest values found for a spiral galaxy. The corotation radius $R_{\mathrm{c}}$ of the bar and the gaseous disc is $R_{\mathrm{c}}=9.8_{-2.0}^{+2.9} \mathrm{kpc}$, implying a ratio $\mathcal{R}=R_{\mathrm{c}} / a_{\mathrm{B}}$ of $2.0_{-03}^{+0.5}$, where $a_{\mathrm{B}}$ is the bar radius. The ratio is well beyond the usual range of values, $1.0 \leq \mathcal{R} \leq 1.4$, found for fast bars of high surface brightness barred galaxies. This implies that the bar in UGC 628 is slow.

Conclusions. As shown through the use of numerical simulations, fast bars survive when the inner mass distribution of galaxies is dominated by the baryons over the dark matter. Our result suggests that the presence of slow bars in galaxies is likely related to the dominance of dark matter over the mass distribution.
\end{abstract}

Key words. galaxies: kinematics and dynamics - galaxies: spiral - galaxies: structure - galaxies: fundamental parameters cosmology: dark matter

\section{Introduction}

In the last decade, estimates of the pattern speed of stellar bars in spiral galaxies have shown that they have high amplitudes (Gerssen et al. 1999; Aguerri et al. 2003; Corsini et al. 2007). As a consequence, the ratio of the corotation radius, $R_{\mathrm{c}}$, to the semi-major axis of the bar, $a_{\mathrm{B}}$, is usually observed in the range $[1 ; 1.4]$, within the uncertainties. A bar whose ratio falls within this range is called a fast bar because its pattern speed cannot be larger, according to theoretical models. A bar that rotates faster would have a smaller corotation radius at a fixed bar semilength $(\mathcal{R}<1)$ and should not exist. Indeed, analytic solutions of the equations of motion and numerical simulations have shown that periodic orbits of stars and gas are aligned perpendicular to the bar in regions beyond corotation and tend to destroy it (Contopoulos \& Papayannopoulos 1980; Athanassoula 1992). The numerical models of Athanassoula (1992) also show simulated bars with $1.0 \leq \mathcal{R} \leq 1.4$, which is in excellent agreement with observations. Conversely, stellar bars having $\mathcal{R}>1.4$ are called slow bars and can exist, though they have not been observed yet.

Classifying a bar as fast or slow thus relies on the measurements of its pattern speed, its extent and on the details of the disc rotation curve. Most pattern speed determinations were made through the application of the Tremaine-Weinberg method (TW; Tremaine \& Weinberg 1984). Starting from the continuity equation, Tremaine $\&$ Weinberg showed that a pattern speed can be defined as $\Omega_{\mathrm{p}} \sin (i)=\int_{-\infty}^{\infty} \Sigma v \mathrm{~d} x / \int_{-\infty}^{\infty} \Sigma x \mathrm{~d} x$ where $i$ is the disc inclination, $\Sigma$ is the surface density of the tracer, $v$ is the

* Figures 2 and 4 are only available in electronic form at http://www . aanda. org line-of-sight velocity and $x$ is the position along slices parallel to the disc major axis. Since the continuity equation underlies this estimate, applying the TW method to the old stellar population is most accurate, as it is expected to satisfy the continuity equation better than the gas. For this reason, astronomers have generally used long-slit spectroscopy of stellar absorption lines of early type barred discs for these estimates (Merrifield \& Kuijken 1995; Aguerri et al. 2003; Gerssen et al. 1999, 2003; Corsini et al. 2003, 2007). Nonetheless, the TW method has been applied with some success to the kinematics of the neutral, molecular, and warm ionized gas (Bureau et al. 1999; Rand \& Wallin 2004; Hernandez et al. 2005; Emsellem et al. 2006; Fathi et al. 2007; Beckman et al. 2008; Meidt et al. 2008), though the conditions under which it can be applied to the gas component are not yet fully understood.

In this letter, we estimate the bar pattern speed of UGC 628. This galaxy was selected because its morpological type and dark matter properties strongly differ from those of galaxies usually observed for this measurement. Indeed, UGC 628 is a Magellanic barred, low surface brightness galaxy (LSB, hereafter). Like most galaxies of this kind, its mass is entirely dominated by dark matter at all galactocentric radii (de Blok \& Bosma 2002). Our objective is to investigate how the dynamical properties of a bar in a dark matter dominated galaxy compare with those of bars in more typical, high surface brightness galaxies.

For this measurement, we used optical 3D spectroscopic observations of the ionized gas in UGC 628. It is a difficult task in the emission line gas and virtually impossible in the stellar absorption lines because the emission is very faint. This explains why pattern speeds have predominately been measured in high surface brightness galaxies. 

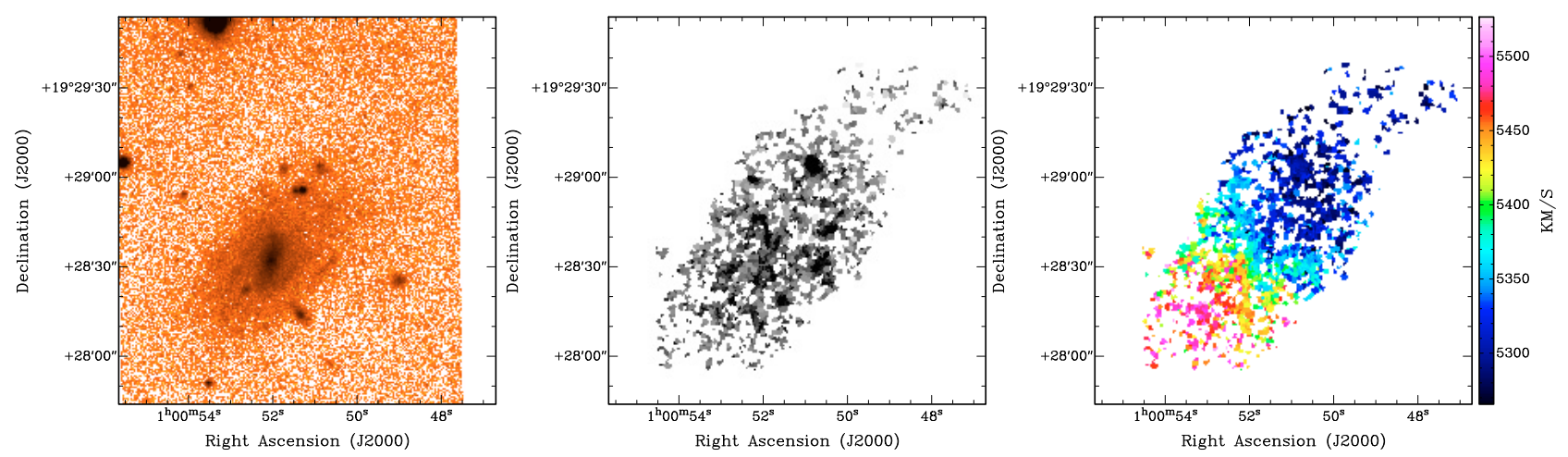

Fig. 1. UGC 628. R-band image (left panel, de Jong 1996), H $\alpha$ integrated emission and velocity maps (middle and right panels, Chemin et al., in preparation). A logarithmic scale is used in left and middle panels.

The kinematical and photometric observations are described in Sect. 1 and their analysis is done in Sect. 3. A discussion and brief concluding remarks are presented in Sect. 4.

\section{Object characteristics and observations}

UGC $628\left(D=71.2 \mathrm{Mpc}, i=56^{\circ}\right)$ was observed with FANTOMM, an optical Fabry-Perot interferometer (Hernandez et al. 2003) mounted on the Canada-France-Hawaii Telescope (CFHT). These observations are part of a large survey of the kinematics of low surface brightness galaxies (see Chemin et al., in preparation for more details). The angular sampling of the $\mathrm{H} \alpha$ datacube is $0^{\prime \prime} .49$ per (square) pixel, corresponding to $\sim 170 \mathrm{pc}$ at the distance of the galaxy. The resolving power of the etalon is 12450 at the redshifted $\mathrm{H} \alpha$ line. The spectral range of $262.7 \mathrm{~km} \mathrm{~s}^{-1}$ was scanned through 24 channels. The exposure time was 8.1 min per channel. The data reduction procedure is fully described in previous papers (e.g., Chemin et al. 2006). Figure 1 shows a $R$-band image of UGC 628 (de Jong 1996), the distribution and the velocity field of the $\mathrm{H} \alpha$ emission line. The broad band image shows a seemingly undisturbed spiral galaxy with an inner stellar bar aligned approximately North-South. The $\mathrm{H} \alpha$ emission displays a faint disc of diffuse gas. It is brighter in the bar region as well as along the two spiral arms, which are more or less parallel to the major axis of the disc.

\section{Analysis}

\subsection{The bar semi-major axis}

The intrinsic radius of the bar, $a_{\mathrm{B}}$, can be estimated in several ways (Athanassoula \& Misiriotis 2002, and references therein). We use the two methods presented in Wozniak et al. (1995) and Aguerri et al. (2000) to derive $a_{\mathrm{B}}$ for UGC 628.

In the first method, isophotes are fitted to images of the galaxy. We used the BVRI images from de Jong (1996) and derived profiles of ellipticity and position angle, PA, of the isophotal major axis using the ellipse task in IRAF. The PA is defined as the angle between the semi-major axis of the receding half of the velocity field and North (angle calculated North through East). Foreground and clumpy emission regions are masked before fitting. All photometric bands give similar results though the $B$ - and $I$-bands show more scatter which, could be due to the low signal-to-noise ratio of the images. Results given hereafter have been obtained by averaging the values from all photometric bands. Only the results for the $R$-band image are shown for clarity. Wozniak et al. (1995) showed that $a_{\mathrm{B}}$ can be estimated by

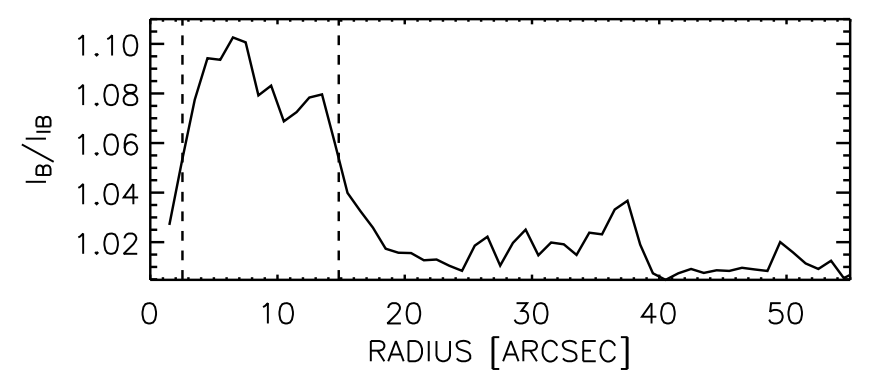

Fig. 3. Fourier analysis of a $R$-band image of the LSB galaxy UGC 628. The radial profile of the bar intensity $I_{\mathrm{B}}$ to the interbar intensity $I_{\mathrm{IB}}$ is shown. The vertical dashed lines indicate the bar region. The outer line at $R=14.8^{\prime \prime}$ corresponds to the deprojected bar semi-major axis.

determining the location of a discontinuity in the distribution of PA and ellipticity with radial distance. The change is such that the regions in the bar show constant PA, which then abruptly changes to the disc PA. Similarly, there is an abrupt change in the ellipticity from a fairly flat distribution to something significantly more round. Figure 2 shows the $R$-band radial profiles of PA and ellipticity of UGC 628 in the central regions of the galaxy. UGC 628 has a very small bulge (Fig. 1) that should not contaminate the profiles significantly. The ellipticity peaks around a projected radius $r=9^{\prime \prime} .4$ before the end of the bar. The bar position angle is constant on the sky plane $\left(\sim 163^{\circ}\right)$ and well separated from the disc orientation $\left(\mathrm{PA}=138^{\circ}\right)$. The projected bar radius is $11^{\prime \prime} .3$, which corresponds to $13^{\prime \prime} .2$ after deprojection.

The second method uses a Fourier decomposition of an image. Aguerri et al. (2000) define the bar as having the radial range where $I_{\mathrm{B}} / I_{\mathrm{IB}}>0.5\left[\max \left(I_{\mathrm{B}} / I_{\mathrm{IB}}\right)-\min \left(I_{\mathrm{B}} / I_{\mathrm{IB}}\right)\right]+$ $\min \left(I_{\mathrm{B}} / I_{\mathrm{IB}}\right)$. In this expression $I_{\mathrm{B}}=I_{0}+I_{2}+I_{4}+I_{6}$ is the bar intensity, with $I_{m}$ being the even Fourier coefficients of order $m$, and $I_{\mathrm{IB}}=I_{0}-I_{2}+I_{4}-I_{6}$ is the interbar intensity. We fitted the Fourier coefficients with a least-squares routine to a deprojected version of the $R$-band image of UGC 628 . The results of the Fourier analysis is shown in Fig. 3. Following the prescription of Aguerri et al. (2000), the bar region extends between $2^{\prime \prime} .5 \leq R \leq 14^{\prime \prime} .8$. The bar radius is the upper limit of this range.

The two bar radius estimates are in very good agreement. The final bar semi-major axis is derived as the average of these two values, which gives $a_{\mathrm{B}}=(14.0 \pm 0.8)^{\prime \prime}$ or $(4.8 \pm 0.3) \mathrm{kpc}$.

\subsection{The bar pattern speed}

We use the $\mathrm{H} \alpha$ velocity field of UGC 628 (Fig. 1 and Sect. 1) with which intensity weighted mean positions and velocities are 
measured along many slices aligned parallel to the kinematical major axis of the galaxy. This is the TW method.

Though reasonable values of pattern speeds are derived using $\mathrm{H} \alpha$ observations (e.g., Beckman et al. 2008), the conditions under which the method is applicable to the warm ionized gas is not entirely clear. However, Hernandez et al. (2005), through the use of $N$-body/SPH simulations, found that the method was robust for the ionized gas but with the proviso that prominent regions where strong gas shocks occur should be avoided. Such regions introduce mean local velocity and position discontinuities that can bias slope measurements used in the TW method. Fortunately, such discontinuites are easily identifiable in the TW diagrams.

The kinematical position angle of the disc $\left(\mathrm{PA}=(139 \pm 3)^{\circ}\right)$ defined the orientation of the slices. It is derived from a tiltedring model of the $\mathrm{H} \alpha$ velocity field (Chemin et al., in prep.). There is very good agreement between the photometric and kinematical major axes (Sect. 3.1). The pattern speed of the bar is taken to be constant as a function of radius so that a single value can be derived using the TW equation of Sect. 1. We refer to Merrifield et al. (2006) and Meidt et al. (2008) for discussions of the radial dependence of pattern speed measurements in galaxies.

In practice, the integrals of $\Sigma v$ and $\Sigma x$ are not determined within ] $-\infty,+\infty$ [ because of the finite extent of the galaxy due to the surface brightness detection limit. The integrals are calculated along slices of maximal aperture $X_{\max }$, with the value of $X_{\max }$ differing from one slice to the next. Results for two apertures values $X_{\text {aperture }}=X_{\max }-\epsilon$ and $X_{\text {aperture }}=X_{\max }$ are shown in Fig. 4, where $\epsilon$ is a small fraction of $X_{\max }$. Most of the integrals have converged (or nearly so) to stable values $\langle X\rangle$ and $\langle V\rangle$ at these two apertures (filled symbols and solid lines). The TW method thus seems to be applicable to the ionized gas data of UGC 628. Integrals that have not converged within $X_{\max }$ are displayed with open symbols and dotted lines. They correspond to the edges of the gas distribution and velocity field, i.e. the most distant regions to the major axis. This is not surprising because the slices in these regions contain very few points.

Figure 5 shows the result of applying the TremaineWeinberg method to UGC 628. Only integrals that have converged within $X_{\text {aperture }}=X_{\max }$ are drawn and kept in the slope fitting of the relationship. The uncertainties in the velocities are typically of the order of 3-4 $\mathrm{km} \mathrm{s}^{-1}$. They depend mainly on the accuracy of the wavelength calibration of the raw datacube. The accuracy on the velocity centroid of an emission line in each pixel is indeed a fraction of a velocity channel, as derived from observations of a reference Neon lamp.

Due to the lopsided galaxy morphology, the average positions are preferentially observed at positive offests. A similar effect has been observed in other $\mathrm{H} \alpha$ or $\mathrm{CO}$ observations (e.g., Rand \& Wallin 2004; Hernandez et al. 2005; Emsellem et al. 2006). As a check for consistency with the stellar tracer, $\langle X\rangle$ values have been derived from the $R$-band image. They have been observed to be offset towards positive values as well.

Only points whose location falls within the bar region $R \leq 14^{\prime \prime}$ in the $2 \mathrm{D} \mathrm{H} \alpha$ image are used to measure the bar pattern speed (filled symbols). The best fit gives $\Omega_{\mathrm{p}}=$ $11.3 \mathrm{~km} \mathrm{~s}^{-1} \mathrm{kpc}^{-1}$. This value does not change when a larger region is used, $R \leq 14^{\prime \prime} .8$, and only increases by $2 \%$ when a smaller region is used, $R \leq 13^{\prime \prime} .2$. All other points lying beyond the bar extent (open symbols) are considered to trace another pattern speed (e.g., that of the spiral arms in the disc). An amplitude of $8.8 \mathrm{~km} \mathrm{~s}^{-1} \mathrm{kpc}^{-1}$ is estimated for them, which is lower than the bar pattern speed.

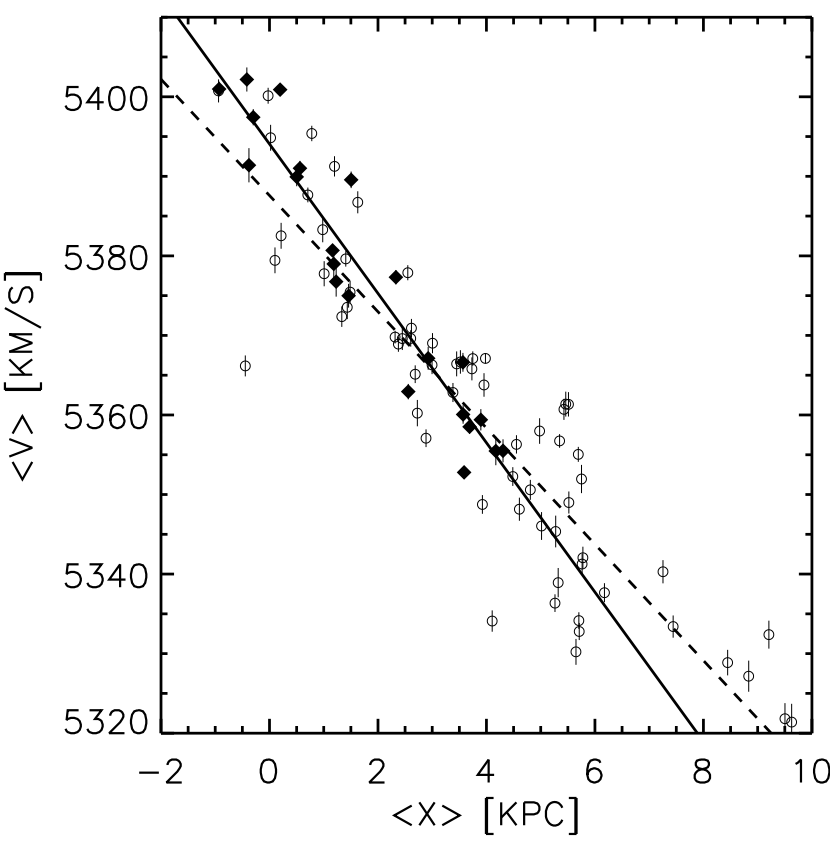

Fig. 5. Bar pattern speed measurement of the LSB galaxy UGC 628. Filled (open) symbols are for data inside (outside, respectively) the bar region. The solid line is the best fitting result to the filled symbols, with slope $\Omega_{\mathrm{p}} \sin (i)$. The dashed line is the best fitting result to the open symbols, with a slope corresponding to the pattern speed of the spiral arms of UGC 628.

The total uncertainty on $\Omega_{\mathrm{p}}$ is obtained by adding in quadrature the uncertainties in $\Omega_{\mathrm{p}}$ due to the uncertainty in the position angle $\left(3^{\circ}\right)$, the inclination $\left(2^{\circ}\right)$, the extent of the region used for fitting $\Omega_{\mathrm{p}}$ (which is the error on the region of influence of the bar, i.e., fixed at the bar radius uncertainty of $0^{\prime \prime} .8$ ) and to the formal $1 \sigma$ uncertainty in the fit. All of the three later uncertainties are of the order of $0.1-0.2 \mathrm{~km} \mathrm{~s}^{-1} \mathrm{kpc}^{-1}$, which is negligible compared to the uncertainty due to the uncertainty in the position angle. $3 \mathrm{D}$ spectroscopy allows us to derive $\Omega_{\mathrm{p}}$ at different position angles (between $136^{\circ}$ and $142^{\circ}$ ) and we found a significant change of $\sim 18 \%$ in $\Omega_{\mathrm{p}}\left(\sim 2.0 \mathrm{~km} \mathrm{~s}^{-1} \mathrm{kpc}^{-1}\right) . \Omega_{\mathrm{p}}$ increases (decreases) towards smaller (larger) position angles than PA $=139^{\circ}$. This is similar to the effect seen by other authors (Debattista 2003; Debattista \& Williams 2004). Because of this dominant source of uncertainty, we adopted $\Omega_{\mathrm{p}}=(11.3 \pm 2.0) \mathrm{km} \mathrm{s}^{-1} \mathrm{kpc}^{-1}$ as the likely best value.

\section{Discussion and conclusion}

Figure 6 (top panel) shows the $\mathrm{H} \alpha$ rotation curve of UGC 628 (Chemin et al., in prep.), derived using a tilted-ring model of the velocity field. The angular velocity profile $\Omega(R)=\frac{V(R)}{R}$ (bottom panel) was used to determine the corotation radius of the bar with the disc $\left(\Omega=\Omega_{\mathrm{p}}\right)$. The corotation radius is $R_{\mathrm{c}}=9.8_{-2.0}^{+2.9} \mathrm{kpc}$ implying a ratio $\mathcal{R}=R_{\mathrm{c}} / a_{\mathrm{B}}=2.0_{-0.3}^{+0.5}$. Given that observed bars having $1.0 \leq \mathcal{R} \leq 1.4$ are defined as being fast (e.g., Gerssen et al. 1999) and larger values of $\mathcal{R}$ correspond to slow bars, UGC 628 appears to host a slowly rotating bar.

The slowest bar found before UGC 628 was for the blue compact dwarf galaxy NGC 2915 having $\mathcal{R}>1.7$ (Bureau et al. 1999). What is surprising here is that the bar-like structure of NGC 2915 is only seen in its neutral hydrogen distribution, not in the distribution of its stellar light. Purely gaseous bars are very rare in galaxies because bars are generally devoid 


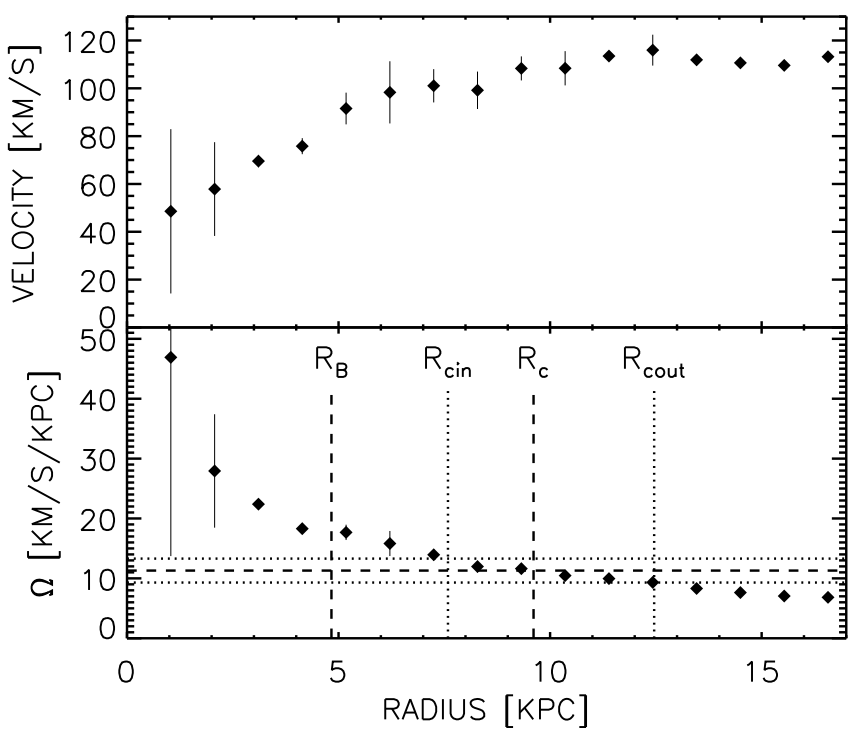

Fig. 6. Rotation curve (top panel) and angular velocity curve (bottom panel) of the LSB galaxy UGC 628. Dashed and dotted horizontal lines are the measured bar pattern speed of $\Omega_{\mathrm{p}}=(11.3 \pm 2.0) \mathrm{km} \mathrm{s}^{-1} \mathrm{kpc}^{-1}$. Dashed and dotted vertical lines respectively indicate the bar semimajor axis $a_{\mathrm{B}}$, the corresponding corotation radius $R_{\mathrm{c}}$ and its lower and upper limits $\left(R_{\text {cin }}, R_{\text {cout }}\right)$ allowed by the uncertainties on $\Omega_{\mathrm{p}}$.

of gas. Apart from that noteable exception, fast bars are always seen in galaxies (Gerssen et al. 1999, 2003; Aguerri et al. 2003; Debattista \& Williams 2004; Corsini et al. 2007). To our knowledge, UGC 628 is thus the first case of an obvious slow stellar bar in a galaxy.

A striking point is that all fast bars are found in normal high surface brightness early-type discs. The stellar mass dominates the dark matter mass in their central regions; their disc is thought to be maximum. It is known that low surface brightness discs are dark matter dominated at all galactocentric distances, even with a realistic mass-to-light ratio for their stellar disc (de Blok $\&$ Bosma 2002). From that, it seems that minimum disc galaxies tend to host slow bars, in opposition to what is observed in maximum disc galaxies. Moreover, the fact that UGC 628 is at the opposite side of the Hubble diagram to the bright galaxies is perhaps the reason why a slow pattern speed is observed for it. With two cases of slow stellar and gaseous bars in UGC 628 and NGC 2915 (respectively), it is thus tempting to argue that the amplitude of bar pattern speeds decreases as a function of the Hubble type. This is in agreement with results from $N$-body simulations (Combes \& Elmegreen 1993).

Other numerical simulations explain how fast bars can be formed and maintained in a high surface brightness galaxy, preferentially within a maximum disc (Debattista \& Sellwood 2000; Valenzuela \& Klypin 2003). These simulations are in agreement with many observations of pattern speeds. Debattista \& Sellwood (2000) argue that barred galaxies with moderately dense halos (like LSBs) should host a slow bar. Our result is therefore in full agreement with their expectation. Other numerical works investigate the bar evolution in LSB discs embedded in cosmological NFW halos (Mayer \& Wadsley 2004). However they do not address the question of the bar pattern speed and the bar length compared with the corotation radius.

Additional works are needed to confirm this new result. First, additional observations of barred low surface brightness discs would help to conclude whether UGC 628 is an isolated case or whether slow bars are common in this type of galaxy. They would also help to investigate whether pattern speeds really decrease as a function of the morphological type. High-resolution HI or optical interferometry like the current Fabry-Perot measurements seems to be the key for the Tremaine-Weinberg method because it is very difficult to observe stellar absorption lines of LSBs and the molecular gas is barely detectable in them (O’Neil \& Schinnerer 2004). A careful analysis of the datacubes of barred LSBs from our sample will be done.

Additional numerical simulations would also be very helpful to investigate the properties of bars in low surface density, dark matter dominated galaxies. Low mass surface density discs are responsible of the cusp-core controversy (de Blok \& Bosma 2002; Hayashi et al. 2007). Kinematical observations of LSBs indeed show that the dark halo seems to have a constant density in its core while steeper density profiles are expected in Cold Dark Matter numerical simulations (e.g. Navarro et al. 1997). How would the pattern speed of a bar embedded within a cusp compare with that of a bar embedded within a core halo? Such simulations could perhaps help in constraining the shape of the inner density profile and alleviate the cusp-core controversy.

Acknowledgements. We are very grateful to P. Amram, C. Balkowski and C. Carignan for their long term support of this project and to M. D. Lehnert for fruitful discussions and a careful reading of the manuscript. We acknowledge the anonymous referee for very constructive remarks. This work is based on observations collected at the Canada-France-Hawaii Telescope, which is operated by the National Research Council of Canada, the Centre National de la Recherche Scientifique de France and the University of Hawaii. O.H. thanks the Fond Québécois de la Recherche sur la Nature et les Technologies and the National Research Council of Canada for their support.

\section{References}

Aguerri, J. A. L., Muñoz-Tuñón, C., Varela, A. M., \& Prieto, M. 2000, A\&A, 361,841

Aguerri, J. A. L., Debattista, V. P., \& Corsini, E. M. 2003, MNRAS, 338, 465 Athanassoula, E. 1992, MNRAS, 259, 345

Athanassoula, E., \& Misiriotis, A. 2002, MNRAS, 530, 35

Beckman, J., Fathi, K., Piñol, N., et al. 2008, Proceedings of the Padova Workshop, Pattern Speeds along the Hubble Sequence, ed. E. M. Corsini, \& V. Debattista [arXiv: 0810.3996]

Bureau, M., Freeman, K. C., Pfitzner, D. W., \& Meurer, G. R. 1999, AJ, 118, 2158

Chemin, L. 2006, MNRAS, 366, 812

Combes, F., \& Elmegreen, B. G. 1993, A\&A, 271, 391

Contopoulos, G., \& Papayannopoulos, T. 1980, A\&A, 92, 33

Corsini, E. M., Debattista, V. P., \& Aguerri, J. A. L. 2003, ApJ, 599, L29

Corsini, E. M., Aguerri, J. A. L., Debattista, V. P., et al. 2007, ApJ, 659, L121 Debattista, V. P. 2003, MNRAS, 342, 1194

Debattista, V. P., \& Sellwood, J. A. 2000, ApJ, 543, 704 Debattista, V. P., \& Williams, T. B. 2004, ApJ, 605, 714 de Blok, W. J. G., \& Bosma, A. 2002, A\&A, 385, 816 de Jong, R. S. 1996, JAD, 2,

Emsellem, E., Fathi, K., Wozniak, H., et al. 2006, MNRAS, 365, 367 Fathi, K., Toonen, S., Falcón-Barroso, J., et al. 2007, ApJ, 667, L137 Gerssen, J., Kuijken, K., \& Merrifield, M. R. 1999, MNRAS, 306, 926 Gerssen, J., Kuijken, K., \& Merrifield, M. R. 2003, MNRAS, 345, 261 Hayashi, E., Navarro, J. F. N., \& Springel, V. 2007, MNRAS, 377, 50 Hernandez, O., Gach, J.-L., Carignan, C., \& Boulesteix, J. 2003, SPIE, 4841, 1472

Hernandez, O., Wozniak, H., Carignan, C., et al. 2005, ApJ, 632, 253

Mayer, L., \& Wadsley, J. 2004, MNRAS, 347, 277

Meidt, S. E., Rand, R. J., Merrifield, M. R., Debattista, V. P., \& Shen, J. 2008, ApJ, 676, 899

Merrifield, M. R., \& Kuijken, K. 1995, MNRAS, 274, 933

Merrifield, M. R., Rand, R. J., \& Meidt, S. E. 2006, MNRAS, 366, L17

Navarro, J. F. N., Frenk, C. S., \& White, S. D. M. 1997, ApJ, 490, 493

O’Neil, K., \& Schinnerer, E. 2004, ApJ, 615, L109

Rand, R. J., \& Wallin, J. F. 2004, ApJ, 614, 142

Tremaine, S., \& Weinberg, M. D. 1984, ApJ, 282, L5

Valenzuela, O., \& Klypin, A. 2003, MNRAS, 345, 406

Wozniak, H., Friedli, D., Martinet, L., Martin, P., \& Bratschi, P. 1995, A\&AS, 111,115 
L. Chemin and O. Hernandez: A slow bar in a dark matter dominated galaxy, Online Material p 1

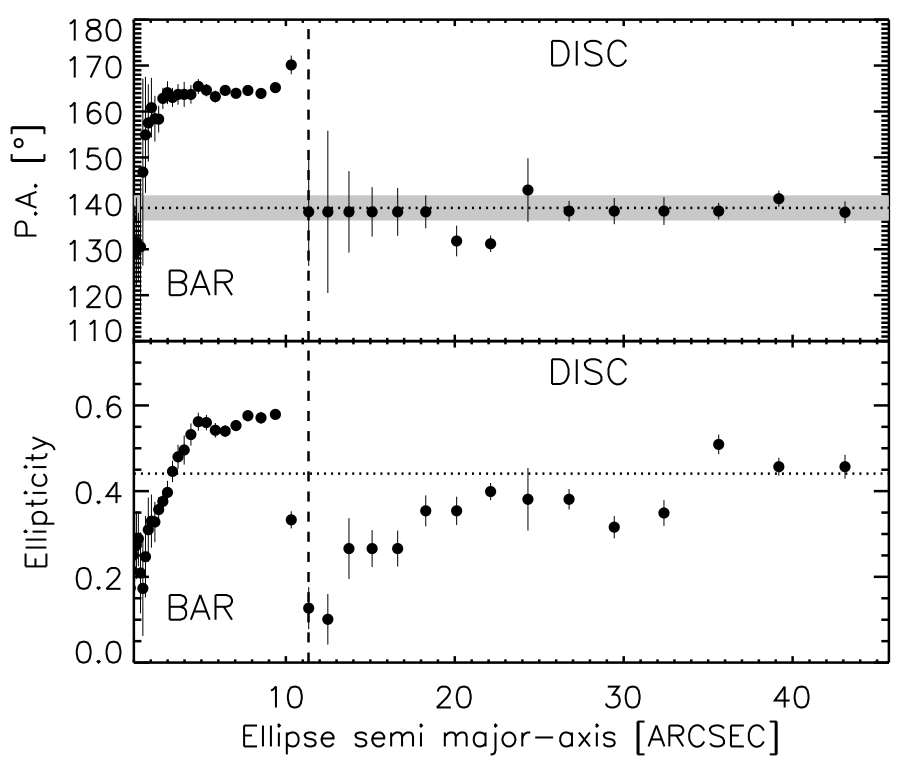

Fig. 2. Results of isophotal ellipse fitting of the $R$-band image of UGC 628. The position angle and ellipticity of the isophotes are shown on top and bottom panels respectively as a function on the projected radius. The dashed vertical line indicates the projected bar semi-major axis. The shaded area represents the kinematic major axis of the disc at $\mathrm{PA}=(139 \pm 3)^{\circ}$. The horizontal line in the bottom panel is the ellipticity of the disc for an inclination of $56^{\circ}$.

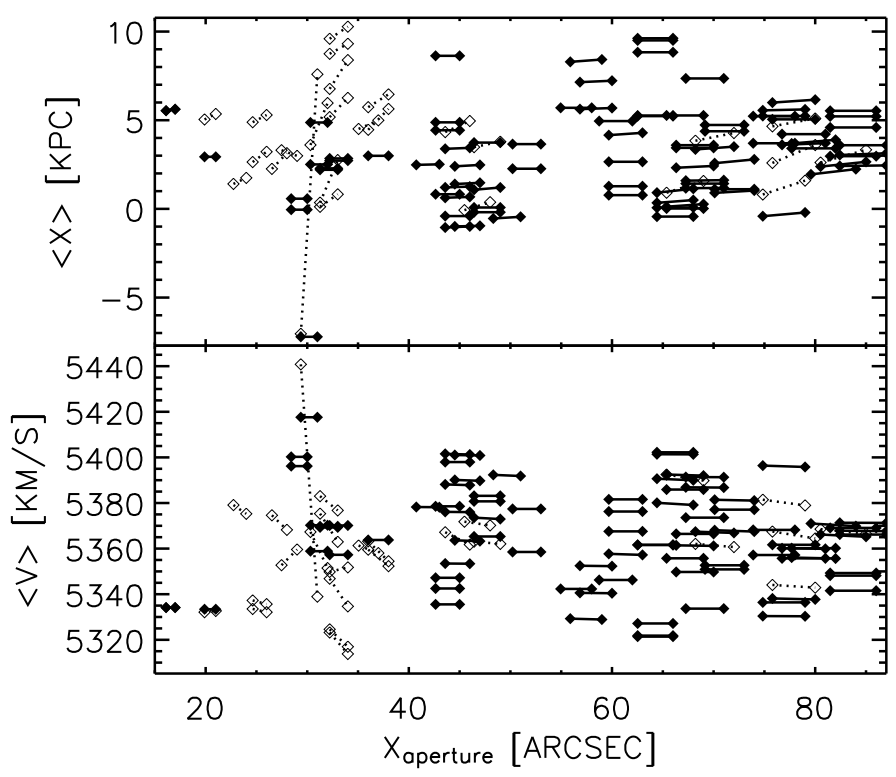

Fig. 4. Tremaine-Weinberg integrals as a function of the aperture length (or extent of the domain of integration). Filled (respectively, open) symbols and solid (dotted) lines are for the integrals that have (have not) converged to stable values. 